\title{
Pore Growth in a Planar Liquid Membrane
}

\author{
A. A. Nepomnyashchy ${ }^{1,2 *}$, V. A. Volpert ${ }^{2}$ \\ ${ }^{1}$ Department of Mathematics and Minerva Center \\ for Nonlinear Physics of Complex Systems, \\ Technion- Israel Institute of Technology, Haifa 32000 Israel \\ 2 Department of Engineering Sciences and Applied Mathematics, \\ Northwestern University, Evanston, IL 60208-3125, USA
}

\begin{abstract}
We study the pore dynamics in a stretched membrane, which is considered as a two-dimensional viscous medium surrounded by a three-dimensional ambient viscous liquid. A closed equation for the pore radius is derived and investigated.
\end{abstract}

Keywords and phrases: pore growth, thin film, liquid membrane, drug delivery

Mathematics Subject Classification: 76D27, 76A20, 62P10

\section{Introduction}

It is known that lipid bilayer membranes, while permeable for water and small molecules, are not permeable for larger molecules. This property of lipid bilayer membranes allows one to use lipid vesicles (liposomes) for transportation of toxic drugs (see, e.g., [1]). When the liposome reaches the target, it is necessary to release the drug. This can be achieved by creating pores in the membrane. The pores appear in a stretched membrane due to thermal fluctuations, similarly to pores in thin liquid films $[2,3]$. In order to enhance the creation of pores, various methods are used, such as applying heat, light, ultrasound, electric field (electroporation) or using acids and proteins [4-7]. The pores in membrane demonstrate nontrivial dynamical behavior $[8,9]$, which so far has been studied only by using simplified models. Our goal is to investigate the dynamics of pores by means of self-consistent mathematical modeling.

In the present paper, we consider the growth of a pore in a stretched planar membrane. In Section 2 , we model the membrane as a viscous incompressible liquid film of finite thickness. In Section 3, we formulate the problem in the case of a microscopically thin membrane, which turns out to be equivalent to that of a viscous film. Section 4 is concerned with the effect of viscous flow of the liquid that surrounds the membrane and Section 5 contains some concluding remarks.

\section{Pore growth in a viscous liquid film with free boundaries}

As a first step, we consider a film of a viscous liquid of thickness $d$ that has viscosity $\eta$ and surface tension $\sigma$. This approach has been previously applied to study pore dynamics in polymer films [3] and liquid membranes [9].

\footnotetext{
*Corresponding author. E-mail: nepom@math.technion.ac.il
} 
It is known that a pore in a film with free boundaries grows exponentially with a certain growth rate $1 / \tau[3]$. Though the functional dependence of the growth rate on the relevant parameters is clear from the dimensional analysis (under the conditions that the inertial effects can be ignored and the density of the liquid $\rho$ is irrelevant),

$$
\tau=k \cdot \frac{\eta d}{\sigma}
$$

where $k$ is a non-dimensional coefficient, the value of this coefficient is a subject of certain controversy. Different values of $k$ are suggested in the basic works on this subject [3,9].

Let us consider an infinite layer of viscous liquid with a round pore of the radius $R(t)$ inside. A cylindrical system of coordinates with the origin at the center of the pore is used. The flow is assumed to be axisymmetric and parallel to the boundaries of the layer $z= \pm d / 2$, i.e., only the radial component of the velocity $v_{r}(r)$ does not vanish. The problem is governed by the continuity equation

$$
\frac{1}{r} \frac{\partial\left(r v_{r}\right)}{\partial r}=0
$$

and the $r$-component of the Stokes equation,

$$
-\frac{\partial p}{\partial r}+\eta \frac{\partial}{\partial r}\left[\frac{1}{r} \frac{\partial}{\partial r}\left(r v_{r}\right)\right]=0
$$

At the stress-free pore boundary $r=R(t)$, the normal component of the stress tensor vanishes,

$$
\sigma_{r r}=-p+2 \eta \frac{\partial v_{r}}{\partial r}=0 .
$$

Also, the kinematic boundary condition is valid:

$$
v_{r}(R)=\frac{d R}{d t}
$$

At infinity, i.e., as $r \rightarrow \infty$, the pressure tends to a fixed value

$$
p(\infty)=-\sigma / d .
$$

Solving the continuity equation (2.2) with the boundary condition (2.5), we find the distribution of the velocity in the film

$$
v_{r}(r)=R \frac{d R}{d t} \frac{1}{r} .
$$

According to $(2.3),(2.6)$, the pressure is constant,

$$
p(r)=-\sigma / d .
$$

Substituting the solution (2.7), (2.8) into the boundary condition (2.4), we find:

$$
\frac{\sigma}{d}-2 \eta \frac{1}{R(t)} \frac{d R(t)}{d t}=0
$$

Thus, in the framework of the approximation, the pore grows exponentially,

$$
R(t)=R(0) \exp (t / \tau)
$$

where

$$
\tau=\frac{2 \eta d}{\sigma} .
$$

That means that the value of the coefficient $k=2$ used in [9] is correct, while the value $k=1 / 2$ suggested in [3] is not correct. 


\section{Pore growth in a viscous liquid membrane}

Equation (2.9) includes a macroscopic film thickness $d$. The thickness of a bilayer lipid membrane is microscopic, therefore from the macroscopic point of view the membrane is a two-dimensional medium. Thus, strictly speaking, equation (2.9) cannot be used in the case of a membrane.

The dynamic equations governing the motion of a membrane are described, e.g., in the review paper [10]. In the case of an incompressible viscous flow in a planar membrane, the force balance is expressed by the equation

$$
\mathbf{f}=\left(-\partial^{\alpha} p_{2 D}+\eta \nabla^{2} V^{\alpha}\right) \mathbf{t}_{\alpha}
$$

where $\mathbf{t}_{\alpha}, \alpha=1,2$, are orthogonal vectors tangential to the membrane surface, $p$ is the two-dimensional pressure acting as a negative surface tension, $V^{\alpha}, \alpha=1,2$, are the corresponding components of velocity, and $\eta$ is the shear surface viscosity of the two-dimensional liquid. Thus, equation (2.3) is retained with a change of variables. The continuity equation (2.2) is also unchanged. The value of $p_{2 D}$ at infinity, $p_{2 D}(\infty)=-|p(\infty)|$, is determined by the tension applied to the membrane rather than by the physical parameters of the film. The boundary condition (2.4) at the pore boundary $r=R(t)$ is also retained [11]. Thus, the full formulation of the problem is equivalent to that of the liquid film dynamics. As a result, we arrive at the equation

$$
2 \eta \frac{d R}{d t}=|p(\infty)| R
$$

which does not include the thickness of the membrane.

\section{Effect of viscous flow in the surrounding liquid}

In this section we consider the influence of the flow in the surrounding liquid on the growth of a pore in a viscous liquid membrane.

Adopting the assumption of an axisymmetric flow in the film, we obtain the following system of equations for the axisymmetric three-dimensional flow $\left(v_{r}(r, z), v_{z}(r, z), p(r, z)\right)$ in the half-space above the membrane $(z>0)$ :

$$
\begin{gathered}
-\frac{\partial p}{\partial r}+\eta^{\prime}\left\{\frac{\partial}{\partial r}\left[\frac{1}{r} \frac{\partial}{\partial r}\left(r v_{r}\right)\right]+\frac{\partial^{2} v_{r}}{\partial z^{2}}\right\}=0, \\
-\frac{\partial p}{\partial z}+\eta^{\prime}\left[\frac{1}{r} \frac{\partial}{\partial r}\left(r \frac{\partial v_{z}}{\partial r}\right)+\frac{\partial^{2} v_{z}}{\partial z^{2}}\right]=0, \\
\frac{1}{r} \frac{\partial}{\partial r}\left(r v_{r}\right)+\frac{\partial v_{z}}{\partial z}=0
\end{gathered}
$$

where $\eta^{\prime}$ is the viscosity of the surrounding fluid. The same equations are valid in the region $z<0$. At the membrane $(r>R)$, the velocity field in the surrounding liquid has to match the surface velocity of the film,

$$
v_{r}(r, 0)=\frac{1}{r} R \dot{R}, \quad v_{z}(r, 0)=0,
$$

where $\dot{R}=d R / d t$. At the pore $(0<r<R)$, the symmetry conditions can be imposed. Due to the symmetry of the problem, $v_{z}(r, z)$ is expected to be an odd function of $z$ and $v_{r}(r, z)$ is an even function of $z$. Therefore,

$$
v_{z}(r, 0)=0, \quad \frac{\partial v_{r}}{\partial z}(r, 0)=0 .
$$

All the functions are assumed to be bounded as $z \rightarrow \pm \infty$.

We solve the formulated problem by means of the Hankel transform. Assume

$$
v_{r}(r, z)=\int_{0}^{\infty} V_{r}(k, z) J_{1}(k r) d k, v_{z}(r, z)=\int_{0}^{\infty} V_{z}(k, z) k J_{0}(k r) d k,
$$




$$
p(r, z)=\int_{0}^{\infty} P(k, z) k J_{0}(k r) d k .
$$

Substituting (4.6), (4.7) into equations (4.1)-(4.3) and using the properties of the Bessel functions, we obtain the following system of equations containing derivatives only with respect to $z$ :

$$
\begin{gathered}
\frac{\partial^{2} V_{r}}{\partial z^{2}}-k^{2} V_{r}=-\frac{1}{\eta^{\prime}} k^{2} P \\
\frac{\partial^{2} V_{z}}{\partial z^{2}}-k^{2} V_{z}=\frac{1}{\eta^{\prime}} \frac{\partial P}{\partial z} \\
\frac{\partial V_{z}}{\partial z}+V_{r}=0
\end{gathered}
$$

Consider the region $z>0$. Eliminating $V_{r}$ and $V_{z}$, we find the closed equation for $P$,

$$
\frac{\partial^{2} P}{\partial z^{2}}-k^{2} P=0
$$

The solution of (4.11) bounded in the region $z>0$ is

$$
P(k, z)=\hat{p}(k) e^{-k z} .
$$

Solving (4.8), (4.9), we find:

$$
V_{r}(k, z)=\hat{v}_{r}(k) e^{-k z}+\hat{p} \frac{k}{2 \eta^{\prime}} z e^{-k z}
$$

and

$$
V_{z}(k, z)=\hat{v}_{z}(k) e^{-k z}+\hat{p} \frac{1}{2 \eta^{\prime}} z e^{-k z} .
$$

The continuity equation gives the relation between functions $\hat{v}_{z}(k), \hat{v}_{r}(k)$ and $\hat{p}(k)$,

$$
-k \hat{v}_{z}(k)+\hat{v}_{r}(k)+\frac{1}{2 \eta^{\prime}} \hat{p}(k)=0 .
$$

Therefore, function $\hat{v}_{z}(k)$ can be eliminated, and the functions $v_{r}(r, z), v_{z}(r, z)$ and $p(r, z)$ can be expressed in terms of two unknown functions, $\hat{v}_{r}(k)$ and $\hat{p}(k)$ :

$$
\begin{gathered}
v_{r}(r, z)=\int_{0}^{\infty}\left[\hat{v}_{r}(k)+\frac{k z}{2 \eta^{\prime}} \hat{p}(k)\right] e^{-k z} J_{1}(k r) d k \\
v_{z}(r, z)=\int_{0}^{\infty}\left[\hat{v}_{r}(k)+\frac{k z+1}{2 \eta^{\prime}} \hat{p}(k)\right] e^{-k z} J_{0}(k r) d k, \\
p(r, z)=\int_{0}^{\infty} k \hat{p}(k) e^{-k z} J_{0}(k r) d k
\end{gathered}
$$

Since $v_{z}(r, 0)=0$ for any $r$, we have

$$
\int_{0}^{\infty}\left[\hat{v}_{r}(k)+\frac{1}{2 \eta^{\prime}} \hat{p}(k)\right] J_{0}(k r) d k=0,0<r<\infty .
$$

Using the orthogonality condition for Bessel functions,

$$
\int_{0}^{\infty} r J_{n}(k r) J_{n}(q r) d r=\frac{1}{q} \delta(k-q),
$$


we find that

$$
\int_{0}^{\infty} r d r J_{0}(q r) \int_{0}^{\infty}\left[\hat{v}_{r}(k)+\frac{1}{2 \eta^{\prime}} \hat{p}(k)\right] J_{0}(k r) d k=\frac{1}{q}\left[\hat{v}_{r}(q)+\frac{1}{2 \eta^{\prime}} \hat{p}(q)\right]=0,
$$

hence

$$
\hat{p}(q)=-2 \eta^{\prime} \hat{v}_{r}(q) .
$$

Substituting (4.21) into (4.16)-(4.18), we obtain

$$
\begin{gathered}
v_{r}(r, z)=\int_{0}^{\infty} \hat{v}_{r}(k)(1-k z) e^{-k z} J_{1}(k r) d k, \\
v_{z}(r, z)=-\int_{0}^{\infty} k \hat{v}_{r}(k) z e^{-k z} J_{0}(k r) d k, \\
p(r, z)=-2 \eta^{\prime} \int_{0}^{\infty} k \hat{v}_{r}(k) e^{-k z} J_{0}(k r) d k .
\end{gathered}
$$

Expressions (4.4), (4.5) give the following integral equations for $\hat{v}_{r}(k)$ :

$$
\begin{gathered}
v_{r}(r, 0)=\int_{0}^{\infty} \hat{v}_{r}(k) J_{1}(k r) d k=\frac{R \dot{R}}{r}, r>R, \\
\frac{\partial v_{r}}{\partial z}(r, 0)=-2 \int_{0}^{\infty} k \hat{v}_{r}(k) J_{1}(k r) d k=0,0<r<R,
\end{gathered}
$$

which are satisfied by the function

$$
\hat{v}_{r}(k)=\frac{\dot{R}}{k} \sin k R .
$$

Substituting (4.27) into (4.22), (4.23), we find the following fields of the velocity components in the surrounding liquid:

$$
\begin{gathered}
v_{z}(r, z)=-\frac{\dot{R} z}{Q} \sqrt{Q-\left(r^{2}+z^{2}-R^{2}\right)}, \\
v_{r}(r, z)=\frac{\dot{R}}{r}\left[R-\frac{1}{\sqrt{2}} \frac{\left(R^{2}-r^{2}\right)^{2}+z^{2}\left(R^{2}+r^{2}\right)+Q\left(R^{2}-r^{2}\right)}{Q \sqrt{Q-\left(r^{2}+z^{2}-R^{2}\right)}}\right], \\
Q=\sqrt{\left(r^{2}+z^{2}-R^{2}\right)^{2}+4 R^{2} z^{2}}, z>0 .
\end{gathered}
$$

Due to the symmetry of the problem,

$$
v_{z}(r,-z)=-v_{z}(r, z), v_{r}(r,-z)=v_{r}(r, z) .
$$

Let us consider now the influence of the flow in the surrounding liquid on the flow in the membrane. Following [10], we can present the balance of forces in the form

$$
\hat{\mathbf{n}} \cdot\left(\boldsymbol{\sigma}^{+}{ }_{-} \boldsymbol{\sigma}^{-}\right)+\mathbf{f}=0,
$$

where $\sigma^{ \pm}$is the three-dimensional stress in the surrounding liquid evaluated at both sides of the membrane surface, $\hat{\mathbf{n}}$ is the unit normal vector to the membrane surface, and $\mathbf{f}$ is determined by formula (3.1). In our case, equation (4.30) is reduced to the following one:

$$
-\frac{\partial p_{2 D}}{\partial r}+\eta \frac{\partial}{\partial r}\left[\frac{1}{r} \frac{\partial}{\partial r}\left(r V_{r}\right)\right]+\tau^{+}-\tau^{-}=0
$$


where

$$
\tau^{ \pm}=\left.\eta^{\prime} \frac{\partial v_{r}}{\partial z}\right|_{z=0 \pm}
$$

The tangential stress on the top membrane surface created by the surrounding liquid flow is

$$
\begin{gathered}
\tau^{+}(r)=-2 \eta^{\prime} \int_{0}^{\infty} k \hat{v}_{r}(k) J_{1}(k r) d k= \\
-2 \eta^{\prime} \dot{R} \int_{0}^{\infty} \sin k R \cdot J_{1}(k r) d k=-2 \eta^{\prime} \frac{R \dot{R}}{r \sqrt{r^{2}-R^{2}}}, \quad r>R ;
\end{gathered}
$$

due to the symmetry, the stress on the bottom membrane surface

$$
\tau^{-}(r)=-\tau^{+}(r)
$$

Taking into account (2.2), we find:

$$
-\frac{\partial p_{2 D}}{\partial r}+2 \tau^{+}(r)=0
$$

Thus,

$$
p_{2 D}(r)=p(\infty)-2 \int_{r}^{\infty} \tau^{+}(\rho) d \rho=0
$$

so that

$$
p_{2 D}(R)=p(\infty)+4 \eta^{\prime} R \dot{R} \int_{R}^{\infty} \frac{d \rho}{\rho \sqrt{\rho^{2}-R^{2}}} .
$$

Substituting $p(\infty)=-|p(\infty)|$ and calculating the integral, we find

$$
p_{2 D}(R)=-|p(\infty)|+2 \pi \eta^{\prime} \dot{R} .
$$

Therefore, the balance equation (2.9) is modified as

$$
2 \eta \frac{\dot{R}}{R}+2 \pi \eta^{\prime} \dot{R}=|p(\infty)|
$$

Integration of equation (4.39) gives

$$
2 \eta \ln (R(t) / R(0))+2 \pi \eta^{\prime}(R(t)-R(0))=|p(\infty)| t .
$$

One can see that the exponential growth of the pore is replaced with time by the linear growth.

Note that equation (A.1) suggested in [9] for the description of the influence of the surrounding fluid based on scaling arguments, is qualitatively similar to (4.39) (with $\left|p_{\infty}\right|=\sigma$ and $\eta=\eta_{2} d$, where $\eta_{2}$ is the volume viscosity of the membrane), up to coefficient $2 \pi$ that could not be found by the dimensional analysis.

\section{Conclusions}

We have considered the pore growth in a planar stretched liquid membrane in the cases of Newtonian rheology of the two-dimensional liquid. Unlike previous works based mostly on dimensional analysis and physical estimates, we have formulated and solved some boundary value problems based on self-consistent mathematical models. We have found that the predictions of a "naïve" approach, which presents the membrane as a film of three-dimensional liquid, are compatible with those obtained in the framework of the more appropriate model of two-dimensional liquid. In the framework of the viscous model, an evolution equation for the pore radius has been derived. It is found that it has the structure suggested 
in the previous works, but solving the boundary value problems allows to find the coefficients in that equation.

It has to be emphasized that the results obtained in the case of a planar viscous membrane cannot be directly applied to pores in real liposomes because of the following reasons. The Newtonian rheology, which ignores the elastic properties of the lipid bilayers, cannot be applied for description of membranes coated by biocompatible polymers [12] and/or membranes that include such substances as hydrogenated soybean oil or cholesterol [13], [14]. A real liposome has a finite size and entraps a liquid inside, hence its stretching is limited, and the pore dynamics is influenced by the layer bending elasticity and the liquid flow through the pore. Also, the model presented above ignores the motion of the liposome with the surrounding liquid, e.g., induced by ultrasound pressure [15], as well as multiplicity of pores. However, these necessary extensions go far beyond the scope of the present paper, and they are left for future research.

Acknowledgements. The work has been partially supported by the US-Israel Binational Science Foundation (grant No. 2008122). V.A.V. gratefully acknowledges the support of NSF grant DMS 1108624.

\section{References}

[1] O.G. Mouritsen, Lipids, curvature, and nano-medicine, Eur. J. Lipid Sci. Technol., 113 (2011), 1174-1187.

[2] B.V. Deryagin, Y.V. Gutop, Theory of the breakdown (rupture) of free films, Colloid J., 24 (1962), 370-374

[3] G. Debrégeas, P. Martin, F. Brochard-Wyart, Viscous bursting of suspended films, Phys. Rev. Lett., 75 (1995), 38863889.

[4] T.L. Andresen, S.S. Jensen, K. Jorgensen, Advanced strategies in liposomal cancer therapy: problems and prospects of active and tumor specific drug release, Progr. Lipid Res., 44 (2005), 68-97.

[5] H. Jespersen, J.H. Andersen, H.J. Ditzel, O.G. Mouritsen, Lipids, curvature stress, and the action of lipid prodrugs: Free fatty acids and lysolipid enhancement of drug transport across liposomal membranes, Biochimie, 94 (2012), 2-10.

[6] G. Enden, A. Schroeder, A mathematical model of drug release from liposomes by low frequency ultrasound, Ann. Biomed. Eng., 37 (2009), 2640-2645.

[7] H. Isambert, Understanding the electroporation of cells and artificial bilayer membranes, Phys. Rev. Lett., 80 (1998), 3404-3407.

[8] M.A. Idiart, Y. Levin, Rupture of a liposomal vesicle, Phys. Rev. E, 69, (2004) 061922.

[9] F. Brochard-Wyart, P.G. de Gennes, O. Sandre, Transient pores in stretched vesicles: role of leak-out, Physica A, 278 (2000), 32-51.

[10] T.R. Powers, Dynamics of filaments and membranes in a viscous fluid, Rev. Mod. Phys., 82 (2010), $1607-1631$.

[11] Z.C. Tu, Z.C. Ou-Yang, Lipid membranes with free edges, Phys. Rev. E, 68 (2003), 061915.

[12] V.P. Torchilin, Recent advances with liposomes as pharmaceutical carriers, Nature Reviews Drug Discovery, 4 (2005), 145-160.

[13] A. Laouini, C. Jaafar-Maalej, I. Limayem-Blouza et al., Preparation, characterization and application of liposomes: state of art, J. Coll. Sci. Biotechn., 1 (2012), 147-168.

[14] S. Raffy, J. Teissie, Control of lipid membrane stability by cholesterol content, Biophys. J., 76 (1999), 2072-2080.

[15] A. Schroeder, Y. Avnir, S. Weisman et al., Controllong liposomal drug release by low-frequency ultrasound: mechanism and feasibility, Langmuir, 23 (2007), 4019-4025. 